\title{
A NUMERICAL MODEL OF STANDARD TO BLOWOUT JETS
}

\author{
V. Archontis AND A. W. Hood \\ School of Mathematics and Statistics, St. Andrews University, St. Andrews KY169SS, UK; vasilis@mcs.st-and.ac.uk \\ Received 2013 March 28; accepted 2013 April 25; published 2013 May 9
}

\begin{abstract}
We report on three-dimensional (3D) MHD simulations of the formation of jets produced during the emergence and eruption of solar magnetic fields. The interaction between an emerging and an ambient magnetic field in the solar atmosphere leads to (external) reconnection and the formation of "standard" jets with an inverse Y-shaped configuration. Eventually, low-atmosphere (internal) reconnection of sheared fieldlines in the emerging flux region produces an erupting magnetic flux rope and a reconnection jet underneath it. The erupting plasma blows out the ambient field and, moreover, it unwinds as it is ejected into the outer solar atmosphere. The fast emission of the cool material that erupts together with the hot outflows due to external/internal reconnection form a wider "blowout" jet. We show the transition from "standard" to "blowout" jets and report on their 3D structure. The physical plasma properties of the jets are consistent with observational studies.
\end{abstract}

Key words: Sun: activity - Sun: magnetic topology

Online-only material: color figures

\section{INTRODUCTION}

Observations have shown that the emergence of magnetic flux from the solar interior is often associated with the emission of hot X-ray jets (e.g., Shibata et al. 1992a). A "standard" scenario, which has been extensively used to model such jets, was reported by Heyvaerts et al. (1977): a bipolar magnetic structure emerges into a (unipolar) pre-existing magnetic field and reconnects to form hot and fast outflows (jets) that are emitted from the interface between the fields into contact. Certain aspects of these "standard" jets have been demonstrated in numerical simulations (e.g., Shibata et al. 1992b; Yokoyama \& Shibata 1995; Moreno-Insertis et al. 2008).

Recent observational studies (e.g., Moore et al. 2010; Sterling et al. 2010; Liu et al. 2011; Shen et al. 2012) have revealed another type of jet that has, in addition to the X-ray emitting plasma, cooler $\left(10^{4}-10^{5} \mathrm{~K}\right)$ plasma that erupts along the jet. There is evidence that the eruption originates at the base of the jet, possibly through the emergence of sheared magnetic flux. The eruptive chromospheric/transition-region material undergoes an ejective motion (100-300 $\mathrm{km} \mathrm{s}^{-1}$ ), widens the surrounding field, and becomes part of the jet. Due to the blast of plasma that opens the area in which the fast ejecta occur, these jets are known as "blowout" jets (e.g., Moore et al. 2010).

In this Letter, we present three-dimensional (3D) numerical experiments of magnetic flux emergence into a unipolar field area. We show that the interaction between the emerging and ambient field results in a series of dynamic phenomena: the formation of hot "standard" jets, the eruption of magnetic flux toward the upper solar atmosphere, and the onset of the "blowout" jets with co-spatial emission of cool and hot plasma. We report on the physical properties of the jets and their 3D structure, both of which show agreement with the observations.

\section{THE MODEL}

We solve the 3D time-dependent, resistive MHD equations in Cartesian geometry, using the Lare $3 \mathrm{~d}$ code (Arber et al. 2001). Viscous and ohmic heating are considered through shock viscosity and Joule dissipation. Explicit uniform resistivity is included, with the magnetic Reynolds number $10^{-3}$. The initial conditions consist of a background hydrostatic atmosphere. A sub-photospheric layer resides in the range $(-6.8 \mathrm{Mm} \leqslant z<0 \mathrm{Mm})$. The photosphere/chromosphere is represented by a layer at $0 \mathrm{Mm} \leqslant z<2.4 \mathrm{Mm}$, which is isothermal at the beginning and then the temperature increases with height, up to $\approx 4 \times 10^{4} \mathrm{~K}$. The layer at $2.4 \mathrm{Mm} \leqslant z \leqslant 3.7 \mathrm{Mm}$ is mimicking the transition region. The uppermost coronal layer (3.7 Mm $<z \leqslant 47.7 \mathrm{Mm}$ ) is isothermal. We use the same temperature stratification as Archontis \& Hood (2012).

The domain contains a uniform magnetic field in the $x z$-plane, with strength $B_{\mathrm{amb}}=6 \mathrm{G}$ so that plasma $\beta \ll 1$ in the corona and $\beta \gg 1$ in the lower atmosphere. The ambient field is oblique, making an angle of $30^{\circ}$, as measured from the negative $x$-axis. Similar experiments (e.g., Archontis \& Hood 2012) have been carried out to explore the effect of the field strength and the angle of the ambient field on the dynamical evolution of the system. At $t=0$, a horizontal twisted flux tube oriented along the positive $Y$-axis is placed at $z_{0}=1.7 \mathrm{Mm}$ below the photosphere. The tube's field is defined by

$$
B_{y}=B_{0} \exp \left(-r^{2} / R^{2}\right), \quad B_{\phi}=\alpha r B_{y},
$$

where $R=425 \mathrm{~km}$ is the radius of the tube, $r$ is the radial distance from the tube axis $\left(r^{2}=x^{2}+\left(z+z_{0}\right)^{2}\right)$ and $\alpha=$ $0.0023 \mathrm{~km}^{-1}$ is the uniform twist around the axis of the tube. The rising motion of the tube is initiated by applying a density perturbation along its axis that makes the middle part of the tube more buoyant:

$$
\Delta \rho=\left[p_{t}(r) / p(z)\right] \rho(z) \exp \left(-y^{2} / \lambda^{2}\right),
$$

where $p_{t}$ is the pressure within the flux tube and $\lambda$ controls the size of the buoyant part of the tube. We use $\lambda=1.7 \mathrm{Mm}$ and $B_{0}=5.2 \mathrm{kG}$ for the initial field strength of the tube. The numerical domain is $[-27.2,27.2] \times[-27.2,27.2] \times$ $[-6.8,47.7] \mathrm{Mm}$ in the longitudinal $(y)$, transverse $(x)$, and vertical $(z)$ directions, respectively. The grid has 360 nodes in all directions with periodic boundary conditions in $x$ and $y$ and a wave damping zone for $z>45 \mathrm{Mm}$ where fluctuations 

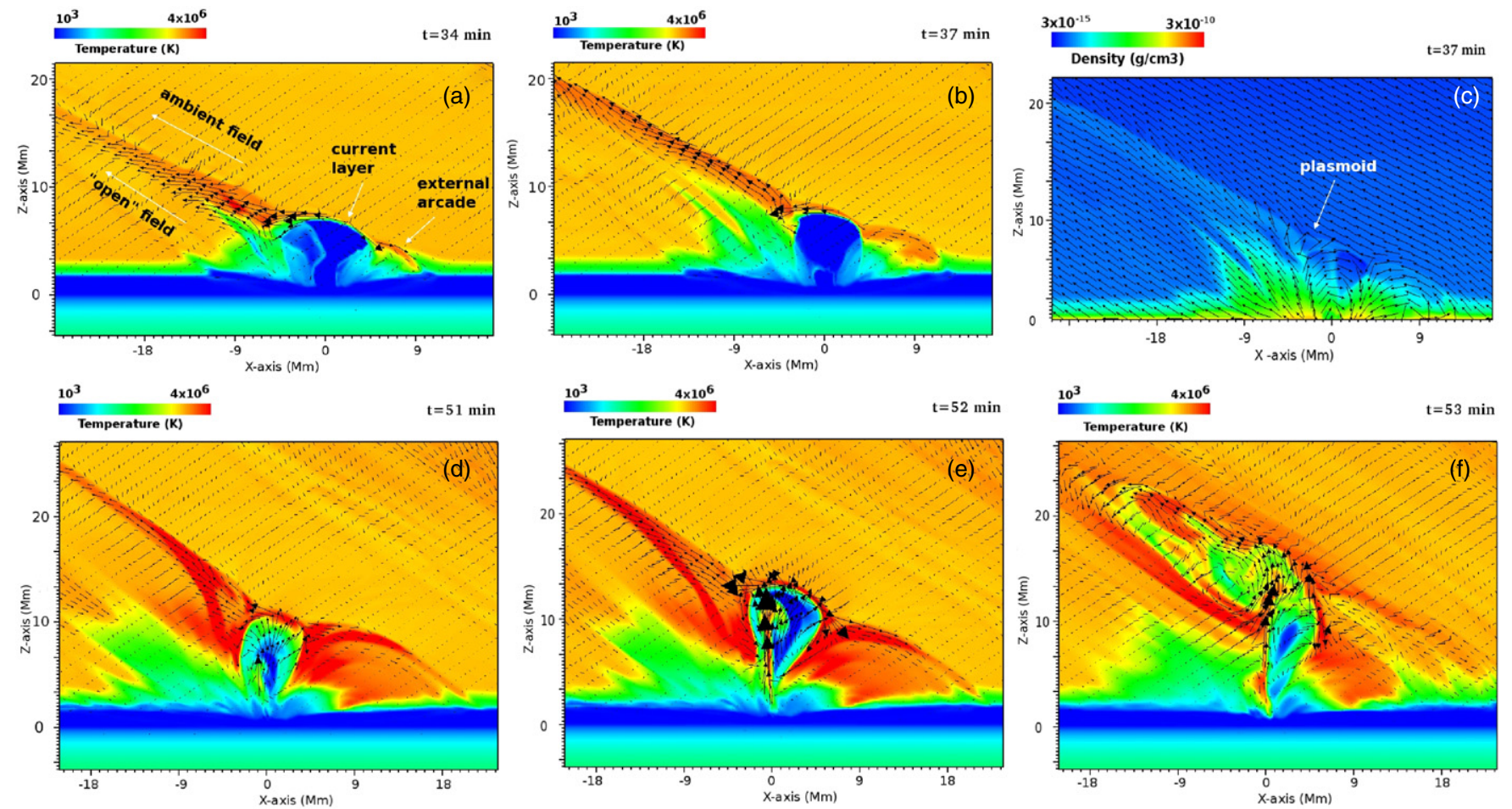

Figure 1. Temperature (panels (a), (b), (d)-(f)) and density (panel (c)) distribution at the vertical midplane. Overplotted (arrows) is the projection of the velocity (panels (a), (b), (d)-(f)) and magnetic (panel (c)) field vector.

(A color version of this figure is available in the online journal.)

of velocity, gas pressure, and density decay exponentially with height.

\section{RESULTS}

Figures 1(a) and (b) show the temperature distribution at the vertical midplane, during the initial stage of the evolution. Overplotted is the projection of the full velocity field vector. The emergence of the field experiences cool adiabatic expansion (blue volume) around the center of the domain. The fieldlines of the emerging (ambient) field are pointing from the negative (positive) to the positive (negative) $x$-axis, resulting in the formation of a current layer at the interface with the ambient field. Reconnection in the current layer generates outflows, which are emitted sideways and collide with the "open" field forming a fast shock. From there, the plasma flows are driven by the pressure gradient force to form a "standard" collimated jet as part of an overall slanted, inverse Y-shaped configuration. The upward jet constitutes the spine of the inverse Y-shaped structure. The jet is filled with hot plasma that reaches temperatures up to $8 \mathrm{mK}$ and speeds that exceeds $250 \mathrm{~km} \mathrm{~s}^{-1}$.

As reconnection at the interface proceeds, the fieldlines rooted in the positive flux region of the emerging field reconnect and form an "open" field domain (see also the white fieldlines in Figure 2(a)). In the negative flux region, reconnecting fieldlines form an external closed arcade-like structure that is heated by the hot plasma of the downward reconnection jet. The top of the arcade is also a flux pile-up regime where the plasma is compressed, increasing the thermal energy of the structure. During the maximum activity, the temperature here can reach values of $10 \mathrm{mK}$. In coronal X-ray images this arcade is typically seen as a "bright point" (e.g., Savcheva et al. 2009). Note that during this initial stage there is no heating at the interior of the emerging volume (blue). Eventually (Figure 1(b)), the arcade becomes wider due to the accumulation of new reconnected fieldlines to it. The hot downward flow of the jet slides on both edges of the arcade, forming the two short legs of the inverse Y-shaped jet. Instead, the upward jet is much more elongated, extending from the reconnection site up to $z \approx 20 \mathrm{Mm}$. The jet and the hot arcade are created almost simultaneously and grow in parallel.

The density distribution in the vertical midplane at $t=$ 37 minutes is displayed in Figure 1(c). It shows that the hot emitted plasma along the spine of the jet is also dense (at least 10 times the density of the background unperturbed plasma). The projected $\mathbf{B}$-field vector demonstrates the direction of the magnetic fieldlines in the different flux domains. The strong current sheet at the interface forms where the fieldlines at the apex of the emerging field come into contact with the ambient field $(x \approx 2 \mathrm{Mm}, z \approx 7 \mathrm{Mm})$. Also note the existence of a "magnetic island" at the left rim of the current sheet, at $x \approx-3 \mathrm{Mm}, z \approx 7 \mathrm{Mm}$. This is a plasmoid (i.e., a small flux rope in 3D), which has been thrown out of the interface current and it is eventually ejected along the reconnected field (e.g., Yokoyama \& Shibata 1995).

Figures 1(d)-(f) show the temperature distribution at a later stage of the evolution. A striking feature is the plasma eruption within the emerging flux region. As in previous experiments (e.g., Archontis \& Török 2008), the eruptive field is a new flux rope formed by the shearing and internal reconnection of the fieldlines along the polarity inversion line. At $t=51$ minutes the center of the cross section of the rising rope is located at $x=0 \mathrm{Mm}, z \approx 7 \mathrm{Mm}$. The flux rope has a strong axial field and it expands as it rises. The embedded plasma comes from the low atmosphere and is dense and cool. Initially, the rope undergoes a slow rising motion. The external reconnection at 

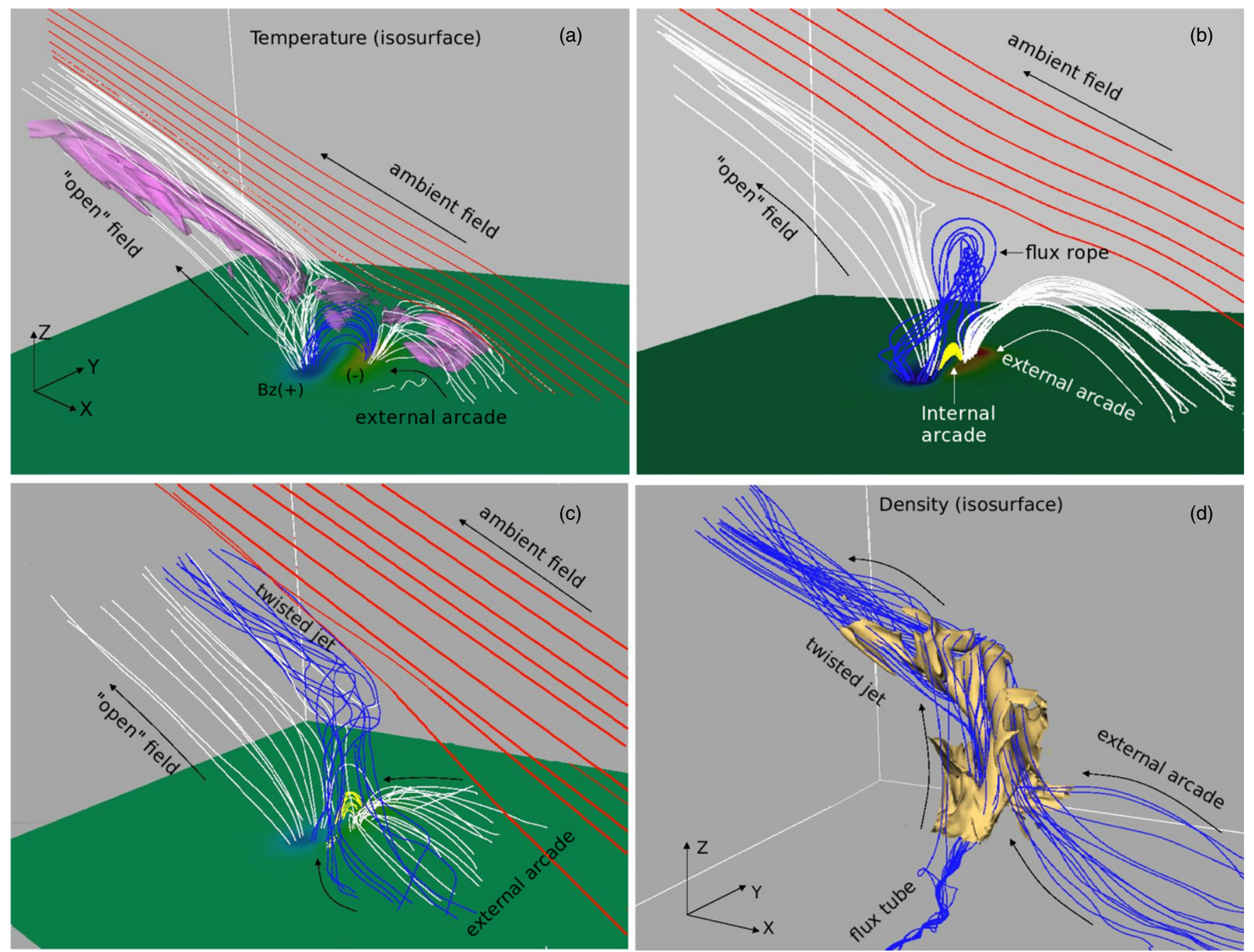

Figure 2. 3D topology of fieldlines during the emission of the jets at $t=37$ minutes (panel (a)), 52 minutes (panel (b)), and 53 minutes (panels (c) and (d)). Isosurfaces represent temperature ( $T \geqslant 3 \mathrm{mK}$; panel (a)) and density $\left(\rho \approx 2 \times 10^{-13} \mathrm{~g} \mathrm{~cm}^{-3}\right.$; panel (d)). The arrows show the direction of the magnetic fieldlines and indicate the various flux domains.

(A color version of this figure is available in the online journal.)

the interface, which persists during emergence, removes flux and downward tension of the outermost (envelope) fieldlines that could halt the ejective eruption of the flux rope. In addition, the rise of the flux rope induces inflows (Figure 1(e)) toward the internal reconnection site underneath it, initiating a tethercutting like mechanism. Both external and internal reconnection lead to the onset of the eruption of the rope. Moreover, the effective internal reconnection creates another reconnection jet (e.g., $z \approx 4.5 \mathrm{Mm}$; Figure $1(\mathrm{e})$ ), which is emitted vertically and helps the flux rope to accelerate. The rapid eruption of the rope brings new flux into contact with the ambient field triggering additional external reconnection. This releases the tension of the outermost fieldlines that envelop the flux rope and, together with the internal reconnection, constitutes an eruptive runaway situation.

During the eruptive phase (Figures 1(e) and (f)), the flux rope does not break-out (vertically) through the ambient flux system but runs along the "open" magnetic field. More precisely, some of the embedded dense plasma erupts into the corona along the spine of the jet, while the rest is ejected toward the external arcade (Figure 1(f)). The eruptive motion broadens the area in which the jet occurs and it blows out the "open" and ambient field in the negative $x$-direction. This new "blowout" jet is now characterized by emission of hot $\left(\approx 10^{7} \mathrm{~K}\right)$ and cool $\left(5-15 \times 10^{4} \mathrm{~K}\right)$ plasma into the corona.

The eruption of the rope, which triggers additional reconnection, has two more important side effects. First, as the rope rises, internal reconnection results in the formation of a new system of reconnecting loops (hereafter, internal arcade) underneath the rope. Second, both arcades grow in size because more fieldlines are attached to them due to reconnection. They also become brighter as the local plasma temperature increases, mainly due to Joule dissipation via reconnection. In our simulations, the internal arcade starts brightening first (with temperatures around $5 \mathrm{mK}$ ), just before the brightening of the external arcade and the onset of the broader spine of the jet. Therefore, the eruption that is (itself) an ejection of cool plasma into the corona is accompanied by heating at the growing arcades and emission of hot plasma into the corona by the external and internal reconnection outflows. The above evolution is consistent with recent observations of blowout jets (e.g., Moore et al. 2010; Sterling et al. 2010).

Figure 2(a) shows the 3D fieldline topology around the "standard" jet at $t=37$ minutes. The selected red fieldlines 

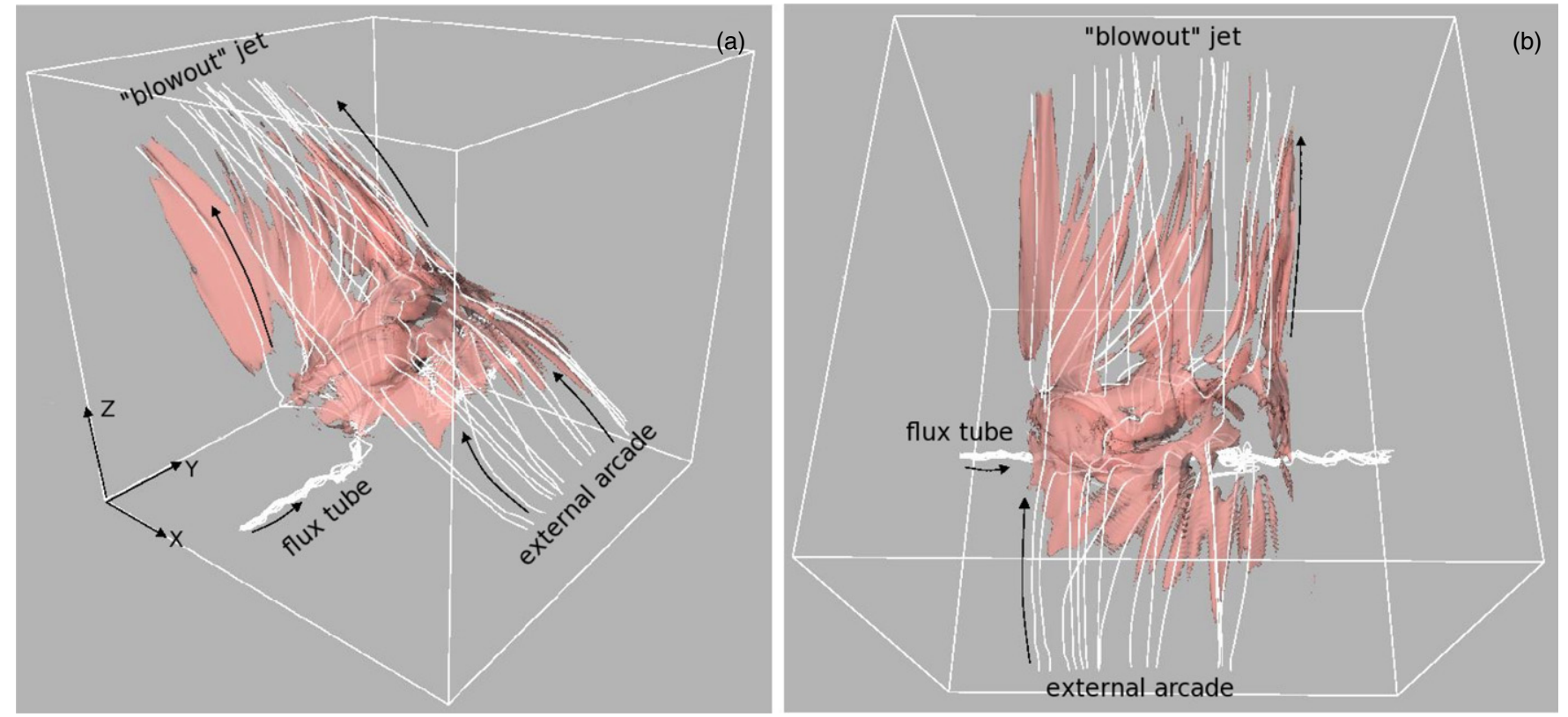

Figure 3. Side-view (panel (a)) and top-view (panel (b)) of the 3D fieldline topology and velocity (isosurface $\geqslant 200 \mathrm{~km} \mathrm{~s}^{-1}$ ) during the blowout jet emission ( $t=54$ minutes). The direction of the fieldlines is shown by the (black) arrows.

(A color version of this figure is available in the online journal.)

represent the ambient magnetic field, just above the emerging field (blue). Reconnected fieldlines (white) show the "open" field on the positive flux region and the external arcade. The horizontal slice shows the $\mathrm{Bz}$ distribution at the photosphere. The isosurface represents temperature (with $T \geqslant 3 \times 10^{6} \mathrm{~K}$ ). The hot plasma region is aligned with the high-speed outflows: it is pronounced along the spine and at the apex of the external arcade. In fact, the large-temperature isosurface delineates the 3D narrow channel of the "standard" jet, which spreads sideways over a limited volume from the interface. However, at a later stage of the evolution, there is a substantial increase to the spatial scale of the jet.

Figure 2(b) shows the fieldline topology at $t=52$ minutes. Now the (blue) twisted fieldlines outline the central part of the erupting flux rope, which expands as it rises. The eruption triggers more reconnection in the vicinity of the expanding plasma. As a result, the (white) fieldlines of the external arcade arch over a longer distance from the negative flux region, which leads to a spatial growth of the arcade. The yellow fieldlines are new reconnected fieldlines that join the opposite polarities of the emerging field and form the internal arcade underneath the flux rope. Note that the (white) fieldlines of the "open" field and the external arcade come closer together in the low-pressure regime underneath the erupting flux rope. Eventually, these fieldlines reconnect, becoming part of the erupting plasma along the jet.

At $t=53$ minutes (Figure 2(c)) the erupting material is ejected along the "open" field. The fieldline topology reveals the twisted nature of the fieldlines along the jet, resulting from the reconnection between the eruptive twisted field and the pre-existing field, which consists of non-twisted oblique fieldlines. Therefore, we are witnessing an untwisting plasma motion during the emission of the blowout jet. At this stage, reconnection also occurs between the "open" field and the external arcade. The reconnected fieldlines (some of them shown in blue) establish links between the two flux systems: on one end they are arching over the dome of the external arcade and on the other they are oriented along the "open" field. Figure 2(d) is a close-up of the twisted field erupting along the jet. The isosurface shows high density $\left(\rho \approx 2 \times 10^{-13} \mathrm{~g} \mathrm{~cm}^{-3}, 100\right.$ times the density in the top corona layer and originally coming from the low atmosphere), which is transported toward corona. The fieldlines have been traced from the isosurface, indicating that the twisted field consists of reconnected fieldlines, joining the jet with the emerging field and the external arcade.

Figure 3 shows the 3D structure of the velocity during the emission of the blowout jet. In contrast to the standard jet (see Figure 2(a)), the blowout jet has a complex structure consisting of many filament-like fast outflows (e.g., Isobe et al. 2005), which jointly form the overall creased veil-shaped configuration of the jet. Also, it is much wider, since the eruption of the field initiates the emission of reconnection outflows over a longer distance, e.g., in the $y$-direction. The fieldlines, which are traced from the velocity isosurface, are reconnected fieldlines that join the emerging field with the external arcade and the "open" field. Note that the different relative orientation of the fieldlines along the "open" field confirms the existence of twist along the upward jet.

\section{DISCUSSION}

In this Letter, we have shown that the dynamical evolution of an emerging field and its interaction with an ambient field in the solar atmosphere triggers the initiation of two types of jets: the "standard" inverted-Y shaped jets and the so-called "blowout" jets. There are two key mechanisms that drive the onset of these jets: reconnection and eruption of magnetic fields. External reconnection between emerging and ambient magnetic field is important to turn on the standard jets, while the eruption of a sheared field within the emerging region and internal reconnection are key processes for the emission of the blowout jets. Our numerical experiments show the transition from one type of jet to the other in a self-consistent manner. 
For the first time, we show the 3D geometry of a blowout jet and report on the differences between the two types of jets concerning their physical properties and fieldline topology. We obtain good agreement between the X-ray, EUV observations (e.g., Moore et al. 2010; Sterling et al. 2010; Liu et al. 2011; Shen et al. 2012), and our numerical results with regard to temperature, density, and velocity of the plasma jets. It is worthwhile to mention that some of the characteristics of the jets (e.g., the maximum height that they reach, their temperature and velocity, etc.) and the eruptions (e.g., whether they are confined or ejective, the exact height and time of the onset, etc.) are parameter-dependent. For instance (e.g., Archontis \& Hood 2012), the change of the relative contact angle and the field strength of the flux systems strongly affects both the efficiency of the external reconnection and also the evolution of the new flux rope within the emerging system. Thus, a detailed quantitative work of the initiation and evolution of the standard and blowout jets requires a full parametric study.

The simulations were performed on the STFC and SRIF funded UKMHD cluster, at the University of St Andrews. The authors are grateful for in-depth discussions during the ISSI workshops: "Magnetic flux emergence in the solar atmosphere" and "Understanding solar jets" in Bern.

\section{REFERENCES}

Arber, T., Longbottom, A., Gerrard, C., \& Milne, A. 2001, JCoPh, 171, 151 Archontis, V., \& Hood, A. W. 2012, A\&A, 537, A62

Archontis, V., \& Török, T. 2008, A\&A, 492, L35

Heyvaerts, J., Priest, E. R., \& Rust, D. M. 1977, ApJ, 216, 123

Isobe, H., Miyagoshi, T., Shibata, K., \& Yokoyama, T. 2005, Natur, 434,478

Liu, C., Deng, N., Liu, R., et al. 2011, ApJL, 735, L18

Moore, R. L., Cirtain, J. W., Sterling, A. C., \& Falconer, D. A. 2010, ApJ, 720,757

Moreno-Insertis, F., Galsgaard, K., \& Ugarte-Urra, I. 2008, ApJL, 673, L211

Savcheva, A., Cirtain, J. W., DeLuca, E. E., \& Golub, L. 2009, ApJL, 702, L32

Shen, Y., Liu, Y., Su, J., \& Deng, Y. 2012, ApJ, 745, 164

Shibata, K., Ishido, Y., Acton, L. W., et al. 1992a, PASJ, 44, L173

Shibata, K., Nozawa, S., \& Matsumoto, R. 1992b, PASJ, 44, 265

Sterling, A. C., Harra, L. K., \& Moore, R. L. 2010, ApJ, 722, 1644

Yokoyama, T., \& Shibata, K. 1995, Natur, 375, 42 
ERRATUM: “A NUMERICAL MODEL OF STANDARD TO BLOWOUT JETS” (2013, ApJL, 769, L21)

V. Archontis AND A. W. Hood

School of Mathematics and Statistics, St. Andrews University, St. Andrews KY169SS, UK

Received 2013 May 17; published 2013 June 10

Due to an error at the publisher, the value of the Reynolds number in the third sentence of the first paragraph of Section 2 is incorrect. The correct sentence should read, "Explicit uniform resistivity is included, with the magnetic Reynolds number $10^{3}$."

IOP Publishing sincerely regrets this error. 